\title{
AUTOMATIC SYMMETRY DETERMINATION AND NORMALIZATION FOR ROTATIONALLY SYMMETRIC 2D SHAPES AND 3D SOLID OBJECTS
}

\author{
Soo-Chang Pei $\dagger$ and Lin-Gwo Liou \\ Department of Electrical Engineering, National Taiwan University, Taipei, Taiwan, Republic of China
}

(Received 27 July 1993; in revised form 30 March 1994; received for publication 12 April 1994)

\begin{abstract}
Determination of the rotational symmetry of a shape is useful for object recognition and shape analysis in computer vision application. However, the usual normalization methods are inapplicable when the shape is $N$-fold rotationally-symmetric $(N>=3)$. In this paper, an efficient and reliable new method using modified Fourier transform is introduced to normalize such a $2 \mathrm{D}$ rotationally-symmetric shape. Furthermore, we extend this new method to the normalization of a $N$-fold axial rotationally-symmetric 3D object. There are two obvious advantages when using this new method: (1) it prevents the high computation load and the difficulty in normalizing the shape's orientation which occurred in the old methods using full matching or correlation functions; and (2) the weakness introduced by the old methods using data reduction (from 2D data to $1 \mathrm{D}$ data) is remedied. Experiments will show the validity of our method.
\end{abstract}

Shape normalization Moment Rotational symmetry Principal axis Fourier transform

\section{INTRODUCTION}

Determination of shape orientation is very useful in a number of computer vision applications, especially for image registration or matching. Before two shapes can be matched for recognition, they are usually scaled and rotated to an identical size and orientation. These pre-processing jobs are usually called $2 \mathrm{D}$ shape normalization.

There are several methods proposed for defining the orientations of 2D shapes, such as the use of principal axes, ${ }^{(1)}$ shape matrices, ${ }^{(2)}$ mirror-symmetry axes, ${ }^{(3-5)}$ etc. However, these methods usually fail when the tested shape is rotationally-symmetric. A shape is said to be rotationally-symmetric (RS) if the shape, after being rotated around its own centroid through any multiple of a certain fixed angle, becomes identical to itself. The reason why they fail is that the principal axes of these RS shapes are undefined, and many RS shapes are themselves not mirror-symmetric.

For defining the orientation of a RS shape, several methods are proposed. Chaudhuri et al. ${ }^{(6)}$ used curvature analysis to determine the best axis of symmetry. A correlation function between pairs of curve points is defined, and determines the best axis of symmetry as the one corresponding to the maximum correlation value. Zahn and Roskies, ${ }^{(7)}$ Wallace and Wintz ${ }^{(8)}$ and Crimmins $^{(9)}$ used the Fourier descriptors to analyze the rotational symmetry of simple planar closed curves. Abu-Mostafa and Psaltis ${ }^{(10)}$ used complex moment $C_{p q}$ s and circular harmonic coefficients $c_{p-q}(r) s$ to analyze $N$-fold RS shapes. After a polar re-sampling, Per-

† Author to whom all correspondence should be addressed. kins $^{(11)}$ used exhaustive matching to determine the order $N$ of rotational symmetry and Highnam ${ }^{(12)}$ reduced the $2 \mathrm{D}$ problem to a linear pattern matching problem. Tsai and $\mathrm{Chou}^{(13)}$ defined the generalized principal axes of a $N$-fold RS shape by using its highorder moment functions. Leou and $\mathrm{Tsai}{ }^{(14)}$ solved the fold number $N$ of a simple RS closed contour by finding the number of crossing points between the average radius and the contour. $\operatorname{Lin}^{(15)}$ utilized the characters about the so-called fold-expanded shape to define the orientation of a $\mathrm{N}$-fold RS shape. $\mathrm{Pei}^{(16)}$ used a modified Fourier descriptor to determine the fold number and then normalizes the orientation of a RS shape.

Generally speaking, a lot of RS shapes cannot be easily represented by only one closed contour. Some RS shapes which have complicated boundary and inside holes belong to this case. Therefore, the methods described in references (6)-(9) and (14) are inapplicable in these situations. Some methods (e.g. reference (11)) attempt to solve this problem in a more general view: they usually re-sample the original $2 \mathrm{D}$ shape in the polar coordinate system $(r-\theta)$, and then make a full matching or calculate the correlation function in the angular direction 0 . Although these methods can determine the fold number of complex RS shapes perfectly, it seems too much computational cost is paid. Besides, the calculated matching or correlation functions contain no information about the shape's orientation, which is not beneficial for the shape normalization. On the other hand, Tsai and his co-workers ${ }^{(13,15)}$ define the orientation of a rotationally-symmetric shape by higher-order moments or fold-expanded shape; their methods do not seem efficient since the fold number 
must be determined before applying their algorithms.

To remedy the above shortages, Pei et al. ${ }^{(16)}$ introduce a method using modified Fourier transform. After a polar re-sampling, the $2 \mathrm{D}$ data function $f(r, \theta)$ is reduced to a $1 \mathrm{D}$ periodic function $g(\theta)$ defined as

$$
g(\theta)=\int_{r=0}^{\infty} f(r, \theta) r \mathrm{~d} r .
$$

Based on the periodicity of $g(\theta)$ of a RS 2D shape, he successfully determines the fold number $N$ and defines the shape's orientation by the $1 \mathrm{D}$ Fourier transform of $g(\theta)$. This method greatly decreases the cost of computation, and the Fourier coefficients of $g(\theta)$ do contain the information of the shape's orientation. However, readers should not forget that the reduction of computational cost resulted from the reduction of input data (2D to 1D). That is to say, some information of periodicity of the shape is lost. For example, some very obvious RS shapes may not be successfully determined because their reduced 1D function $g(\theta)$ s have very weak periodicity (explained in Section 4). Although this situation is not often encountered in real application, there is still a shortage.

In this paper, we try to remedy this shortage and still keep the computational cost low. A modified method using Discrete Fourier transform (DFT) is proposed here. The basic idea of this new method is to reduce the original $2 \mathrm{D}$ continuous data to several $1 \mathrm{D}$ discrete data sequences, not to only one $1 \mathrm{D}$ sequence as before. These 1D sequences are then utilized for the normalization and fold determination of a given RS shape. Furthermore, this modified method is then extended to normalize a $N$-fold axial rotationally-symmetric object in 3D space. A $3 \mathrm{D}$ object $S$ is said to be $N$-fold axial rotationally-symmetric (ARS) if it, after being rotated around a fixed axis $\mathbf{n}$ through any multiple of $2 \pi / N$, becomes identical to itself. After applying the modified method, we can obtain its symmetry axis $\mathrm{n}$, fold number $N$, and its normalized pose. Experiments prove the correctness of the method.

In Section 2, our method is applied to solve the 2D rotational symmetry. In Section 3, our method is extended to solve 3D axial rotational symmetry. Section 4 is the discussion about our method. Section 5 displays several experiments. Section 6 is the final conclusion. The Appendix is about the characters of a $N$-fold ARS 3D object.

\section{TO DETERMINE AND NORMALIZE A $N$-FOLD ROTATIONALLY-SYMMETRIC 2D SHAPE}

\subsection{Define $A \mathrm{~N}$-fold rotationally-symmetric $2 D$ shape.}

A 2D shape $S$ can be denoted by the following signature function $f(x, y)$ defined as

$$
f(x, y)=\left\{\begin{array}{l}
1: \text { if }(x, y) \text { is in } S \\
0: \text { if }(x, y) \text { is not in } S
\end{array}\right.
$$

Without loss of generality, we assume the centroid of the shape $S$ is just located at the origin of the $x-y$ coordinate system.

The shape $S$ is said to be $N$-fold rotationally-symmetric if it, after being rotated through any multiple of $2 \pi / N$, becomes identical to itself(see Fig. 1(a)). That is

$$
f(x, y)=f\left(x^{\prime}, y^{\prime}\right),
$$

where

$$
\left[\begin{array}{l}
x^{\prime} \\
y^{\prime}
\end{array}\right]=\left[\begin{array}{cc}
\cos \theta_{k} & -\sin \theta_{k} \\
\sin \theta_{k} & \cos \theta_{k}
\end{array}\right]\left[\begin{array}{l}
x \\
y
\end{array}\right]
$$

$\left(\theta_{k}=k(2 \pi / N), k\right.$ is an integer $)$.

In order to express the angular periodicity of the RS shape $S$ more explicitly, we may let $f(r, \theta)$ denote the signature of the shape at the location $(r, \theta)$ in the polar coordinate system (see Fig. 1(b)). So equation (3) can be rewritten in a simpler form

$$
f(r, \theta)=f\left(r, \theta+k\left(\frac{2 \pi}{N}\right)\right),
$$

where $x=r \cos \theta ; y=r \sin \theta$.

From equation (5), we know $f(r, \theta)$ is a $1 \mathrm{D}$ periodic function of $\theta$ for any fixed value of $r$.

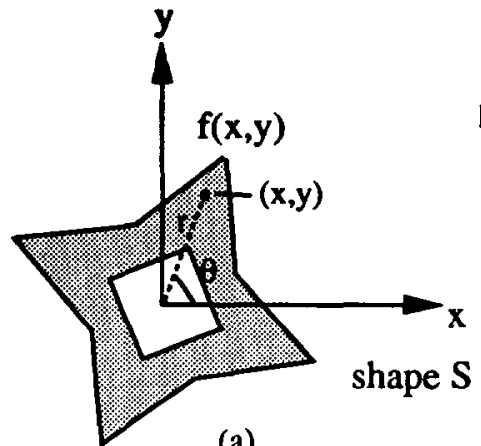

(a)

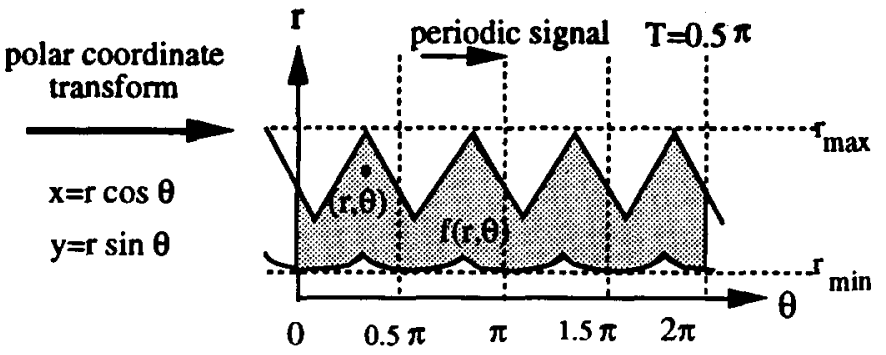

(b)

Fig. 1. (a) An example of a $N$-fold RS shape $S(N=4)$. Transform the original $x-y$ coordinate system to the polar coordinate system for expressing the periodicity of the shape more explicitly. 


\subsection{Fourier transformation of the shape with respect to} $\theta$ only

Let $F(r, \omega)$ denote the 1D Fourier transformation of the shape $S$ with respect to the parameter $\theta$ for any fixed radius $r$. So we have

$$
F(r, \omega) \equiv \int_{\theta=-\infty}^{\infty} f(r, \theta) \mathrm{e}^{-j \omega \theta} \mathrm{d} \theta .
$$

Because $f(r, \theta)$ is a periodic function of $\theta$, it can be expanded by a Fourier series

$$
f(r, \theta)=\sum_{p=-\infty}^{\infty} C_{p}(r) \mathrm{e}^{j p \omega_{0} \theta}
$$

where $\omega_{0} \equiv 2 \pi /(2 \pi / N)=N$ is the fundamental frequency. Its Fourier coefficients, $C_{p}(r)$ s, are then defined as

$$
C_{p}(r) \equiv \frac{N}{2 \pi} \int_{-\pi / N}^{\pi / N} f(r, \theta) \mathrm{e}^{-j p \omega_{0} \theta} \mathrm{d} \theta .
$$

Substituting equation (7) into equation (6), we have

$$
F(r, \omega)=\sum_{p=-\infty}^{\infty} C_{p}(r) \delta\left(\omega-p \omega_{0}\right)
$$

From equation (9), the 1D Fourier transformation $F(r, \omega)$ must be zero except at the frequencies $\omega=p \omega_{0}$ for any fixed $r$. Once the fundamental frequency $\omega_{0}$ of $F(r, \omega)$ can be determined, it is equivalent to obtain the fold number $N$ of the shape. Equations (6)-(9) are the theoretical bases for the methods using Fourier transformation.

\subsection{Our algorithm: finding the fold number $\mathrm{N}$}

For the computational efficiency, we first determine the maximum radius $r_{\max }$ and minimum radius $r_{\min }$ of the given shape (see Fig. 2). The whole shape is contained in a region $A=\left\{(r, \theta) \mid r_{\min } \leq r \leq r_{\max } ; 0 \leq \theta \leq 2 \pi\right\}$.
Without loss of generality, the value of the maximum radius $r_{\max }$ can be set to 1 by properly scaling the given shape.

For the convenient use of digital hardware, the discrete Fourier transform (DFT) should be adopted to analyze the whole problem. So we may divide the whole region $A$ into $M \times N_{s}$ sub-regions (see Fig. 2). The $(m, k)$ th sub-region of $A$ is denoted by $A_{m k}$, where $m=1$ to $M$ and $k=0$ to $N_{s}-1$ ). The two positive integers, $M$ and $N_{s}$, separately represent the radial and angular resolutions. Here we especially call $M$ the section number, and $N_{s}$ the angular sampling number. $A_{m k}$ is defined as

$$
A_{m k} \equiv\left\{(r, 0) \mid r_{m-1} \leq r \leq r_{m} ; \quad \theta_{k} \leq \theta \leq \theta_{k+1}\right\},
$$

where

$$
r_{m} \equiv \sqrt{\left(\frac{m}{M}\right) r_{\max }^{2}+\left(\frac{M-m}{M}\right) r_{\min }^{2}}
$$

and

$$
\theta_{k} \equiv k \frac{2 \pi}{N_{s}}
$$

From the definitions in equations (10)-(12), every subregion $A_{m k}$ is of the same area $\Delta$.

Area of $A_{m k}=\frac{\pi}{N_{s} M}\left(r_{\max }^{2}-r_{\min }^{2}\right) \equiv \Delta$ for all $m, k$

Now, we may define a value $f_{m}(k)$ as follows, which can be considered as a sampling value of the subregion $A_{m k}$

$$
f_{m}(k) \equiv \frac{1}{\Delta} \iint_{A_{m k}} f(r, \theta) r \mathrm{~d} r \mathrm{~d} \theta \approx \frac{2 \pi}{N_{s} \Delta} \int_{r_{m-1}}^{r_{m}} f\left(r, \theta_{k}\right) r \mathrm{~d} r
$$

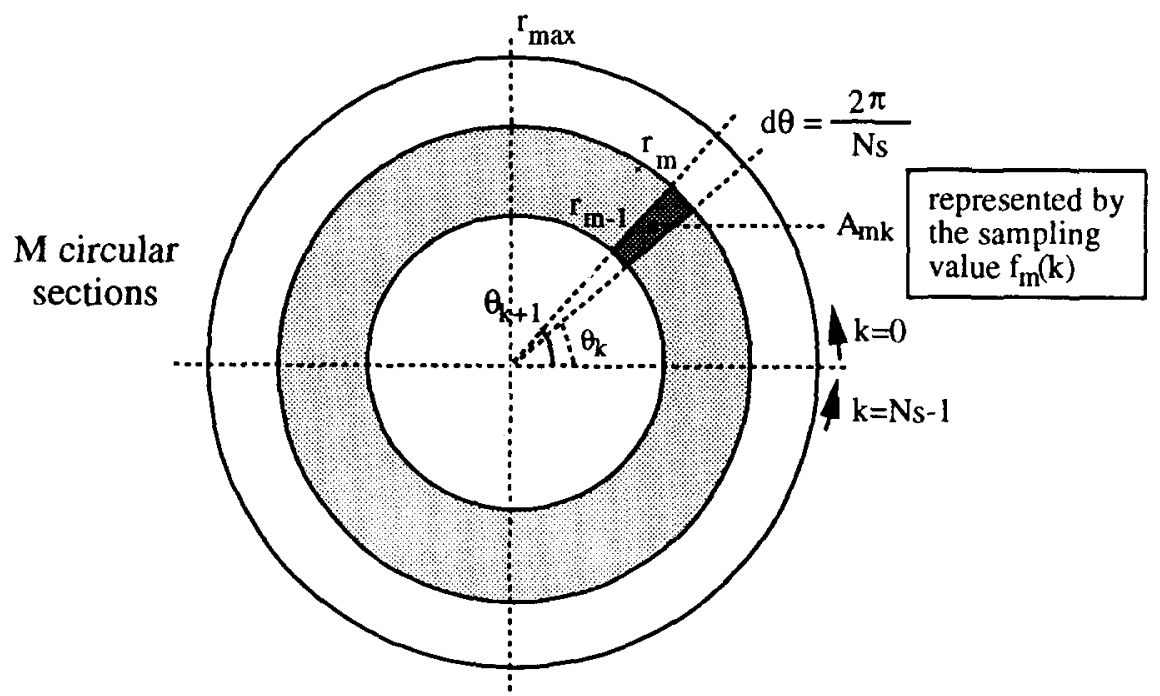

Fig. 2. The area $A$ which contains the whole shape $S$ is divided into $M$ circular sections. The angular sampling number $N s$ uniformly divides each section into $N$ small regions denoted by $A_{m k} s$. The average $f(x, y)$ in the small region $A_{m k}$ is considered as a sampling value $f_{m}(k)$. 
The above equation is approximated because the angular sampling number $N_{s}$ is usually very large (e.g. 256).

Then we will have $M$ 1D sequences $\left\{f_{m}(0), \ldots\right.$, $\left.f_{m}\left(N_{s}-1\right)\right\}(m=1$ to $M)$. The DFT of these sequences can be defined as

$$
F_{m}(k) \equiv \sum_{n=0}^{N_{s}-1} f_{m}(n) e^{-j k\left(2 \pi n / N_{s}\right)}
$$

After substituting equations (7) and (14) into equation (15), we can obtain a new expression for $F_{m}(k)$

$$
F_{m}(k)=\sum_{p=-\infty}^{\infty} T_{m, p} H_{k, p}
$$

where

$H_{k, p} \equiv \frac{1}{N_{s}} \sum_{n=0}^{N_{s}-1} \mathrm{e}^{j(p N-k)\left(2 n \pi / N_{s}\right)} ; \quad T_{m, p} \equiv \frac{2 \pi}{\Delta} \int_{r_{m-1}}^{r_{m}} C_{p}(r) r \mathrm{~d} r$.

From equation (17), it is easy to observe that $H_{k, p}=1$ when $k=p N-a N_{s}$, and $H_{k, p}=0$ for any other $k$ ( $a$ is an integer).

If the angular sampling number $N_{\mathrm{s}}$ happens to be a multiple of the fold number $N, F_{m}(k)$ can be simply written as

$F_{m}(k)= \begin{cases}T_{m, q}+\text { aliasing component } & \text { if } k=q N \\ 0 . & k=\text { otherwise }\end{cases}$

( $q$ is an integer). If $N_{s}$ is not a multiple of $N, F_{m}(k)$ can be simply written as

$F_{m}(k)= \begin{cases}T_{m, q}+\text { aliasing component } & \text { if } k=q N \\ \text { aliasing component } & k=\text { otherwise. }\end{cases}$

Because the sampling number $N_{s}$ is usually very large, the aliasing components in equations (18) and (19) are negligible (see Fig. 3(a)).

Now we may define a pseudo-power spectrum density function $\hat{G}(k)$ as

$$
\hat{G}(k) \equiv \sum_{m=1}^{M}\left|F_{m}(k)\right|^{2} .
$$

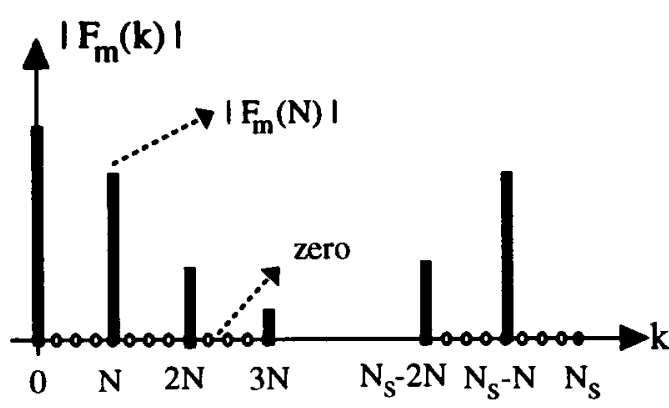

(a)
Detecting the location of the first peak of $\hat{G}(k)$ (i.e. $k=N$, see Fig. 3(b)) is equivalent to determining the fold number $N$ of the given shape. Because the function $\hat{G}(k)$ is used for determining the fold number, it is especially called the $M$-indicator in this paper. Notice the $M=1$ indicator is just the same as the method described in reference (16).

\subsection{Normalization of shape orientation}

After determining the fold number $N$, its corresponding $M$ DFT values, $F_{m}(N)$ s, can be obtained. These values are usually complex numbers. Let's define $C_{1}$ as the sum of these complex values.

$$
C_{1} \equiv \sum_{m=1}^{M} F_{m}(N)=r_{c} \mathrm{e}^{j \theta_{c}}
$$

where $0 \leq \theta_{c} \leq 2 \pi$ and $r_{c}>0$. Notice the angle $\theta_{c}$ will not be affected by the section number $M$ because every sub-region $A_{m k}$ has the same area $\Delta$.

If the original shape $S$ is rotated by an angle $\theta_{s}$, the rotated shape $S^{\prime}$ can be represented by $f\left(r, \theta-\theta_{s}\right)$. We can expand this function by a Fourier series

$$
f\left(r, \theta-\theta_{s}\right)=\sum_{p=-\infty}^{\infty}\left(C_{p}(r) e^{-j p \omega_{0} \theta_{s}}\right) e^{j p \omega_{0} \theta} .
$$

Comparing equation (22) with equations (7) and (17), we know rotating the shape by an angle $\theta_{s}$ will induce a constant phase shift $\exp \left(-j p \omega_{0} \theta_{s}\right)$ to its $p$ th Fourier coefficient $C_{p}(r)$ and $T_{m, p}$. If the aliasing components described in equations (18) and (19) are negligible, an additional constant phase shift $\exp \left(-j N \theta_{\mathrm{s}}\right)$ will be added to all of the $F_{m}(N) \mathrm{s}$, too. Therefore, the orientation information of the RS shape is contained in the complex value $C_{1}$.

If the original shape $S$ can be rotated by an appropriate angle $\theta_{s}$ such that the calculated $C_{1}$ of the new shape is a real positive value (phase $=0$ ), we say that the new rotated shape $\hat{S}$ has been orientation-normalized (see Fig. 4). Such an appropriate rotating angle $\theta_{s}$ is easily calculated by

$$
\theta_{s}=\frac{1}{N} \theta_{c}
$$

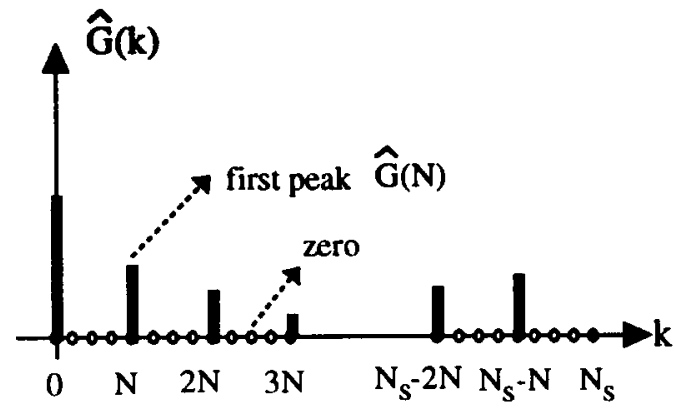

(b)

Fig. 3. (a) The DFT of the 1D data sequence $\left\{f_{m}(k)\right\}$, denoted by $\left\{F_{m}(k)\right\}$. (b) The pseudo power spectrum function $\hat{G}(k) . k=0$ to $N_{s}-1$. If $N_{s}$ is large enough, $F_{m}(k)$ and $\hat{G}(k)$ is zero except at the points $k=q N$. 


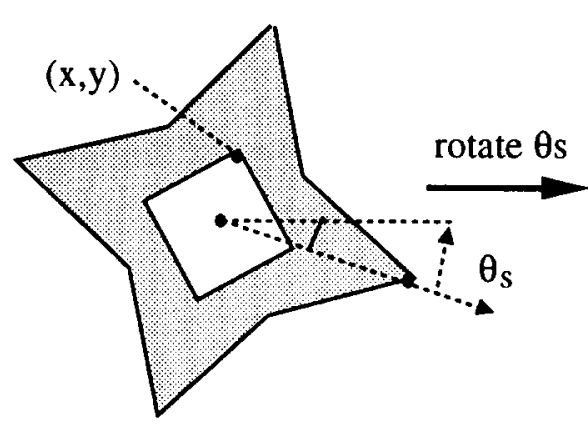

shape $S$

not orientation-normalized

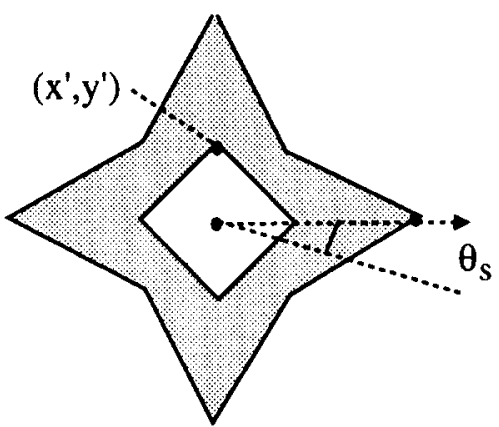

shape $\widehat{S}$

orientation-normalized

Fig. 4. Suitably rotate the original shape $S$ by an angle $\theta_{s}$ to normalize its orientation.

It may cause problems if the amplitude of $C_{1}$ is too small. Because any one of $F_{m}(N)$ s has the same phase shift $\exp \left(-j N \theta_{s}\right)$, we may choose the one (say $\left.F_{i}(N)\right)$ which has the largest amplitude instead of the original $C_{1}$. Therefore,

$$
\theta_{s}=\frac{1}{N}\left(\arg \left(F_{i}(N)\right)\right)
$$

Obviously, the value of the angle $\arg \left(F_{i}(N)\right)$ will be affected by the value of $M$ now.

Now the whole algorithm is completed.

\section{EXTENSION TO A N-FOLD AXIAL ROTATIONALLY-SYMMETRIC 3D OBJECT}

\subsection{Define a $\mathrm{N}$-fold axial rotationally-symmetric object in $3 D$ space}

An object $S^{\prime}$ in $3 \mathrm{D}$ space can be denoted by a signature function $f^{\prime}(X, Y, Z)$. Without loss of generality, we assume the centroid of the object is located at the origin of the $X Y Z$ coordinate system (see Fig. 5(a)).

$$
f^{\prime}(X, Y, Z)=\left\{\begin{array}{l}
1: \text { if }(X, Y, Z) \text { is in } S \\
0: \text { if }(X, Y, Z) \text { is not in } S
\end{array} .\right.
$$

The object $S^{\prime}$ in 3D space is said to be $N$-fold axial rotationally-symmetric (ARS) if it, after being rotated through any multiple of $2 \pi / N$ around a fixed axis $\mathbf{n}$, becomes identical to itself. This axis $\boldsymbol{n}$ is called the symmetry axis. It means

$$
f^{\prime}(X, Y, Z)=f^{\prime}\left(X^{\prime}, Y^{\prime}, Z^{\prime}\right)
$$

where

$$
\begin{aligned}
{\left[\begin{array}{l}
X \\
Y^{\prime} \\
Z^{\prime}
\end{array}\right]=} & {\left[\begin{array}{lll}
\left(n_{x}^{2}-1\right) c+1 & n_{x} n_{y} c-n_{z} s & n_{x} n_{z} c+n_{y} s \\
n_{x} n_{y} c+n_{z} s & \left(n_{y}^{2}-1\right) c+1 & n_{y} n_{z} c-n_{x} s \\
n_{x} n_{z} c-n_{y} s & n_{y} n_{z} c+n_{x} s & \left(n_{z}^{2}-1\right) c+1
\end{array}\right] } \\
& \times\left[\begin{array}{l}
X \\
Y \\
Z
\end{array}\right]
\end{aligned}
$$

$c=1-\cos (2 k \pi / N), s=\sin (2 k \pi / N), \mathbf{n}=\left(n_{x}, n_{y}, n_{z}\right)^{\mathrm{T}}$ is the unit vector representing the rotation axis.

\subsection{Determining the symmetry axis $\boldsymbol{n}$}

In order to determine the symmetry axis $\mathbf{n}$, we first calculate the dispersion matrix $\mathbf{M}$ of the object $S^{\prime}$

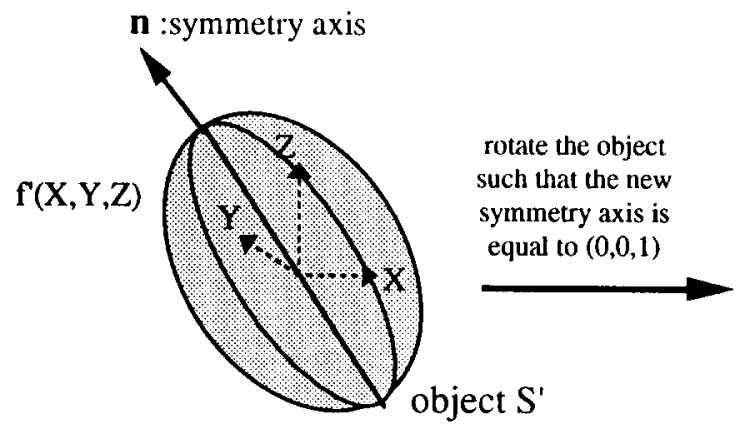

(a)

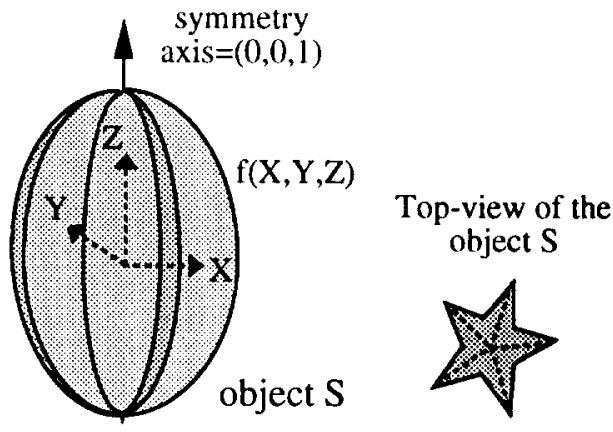

(b)

Fig. 5. (a) An example of a $N$-fold axial ARS 3D object $S^{\prime}$. (N=5, like a five-leaf fruit). Its symmetry axis n can be easily obtained by calculating the dispersion matrix $M$ of the object. (b) Rotate the object $S^{\prime}$ such that the new symmetry axis of the object $S$ is equal to $(0,0,1)$. 
defined as

$$
M \equiv\left[\begin{array}{lll}
m_{200} & m_{110} & m_{101} \\
m_{110} & m_{020} & m_{011} \\
m_{101} & m_{011} & m_{002}
\end{array}\right]
$$

where

$$
m_{i j k}=\frac{1}{|S|^{i+j+k}} \iiint f^{\prime}(X, Y, Z) X^{i} Y^{j} Z^{k} \mathrm{~d} X \mathrm{~d} Y \mathrm{~d} Z,
$$

and $|S|$ is the volume of the object $S$.

The three eigenvalues $\left\{\lambda_{1}, \lambda_{2}, \lambda_{3}\right\}$ and their corresponding eigenvectors $\left\{\mathbf{v}_{1}, \mathbf{v}_{2}, \mathbf{v}_{3}\right\}$ of the dispersion matrix $M$ can be easily calculated. However, to a $N$-fold ARS object, two of the three eigenvalues must be equal (e.g. $\lambda_{1}=\lambda_{2}$ ); and the corresponding eigenvector $v_{3}$ of the distinct eigenvalue $\lambda_{3}$ is just the desired symmetry axis $\mathbf{n}$. The proof of the above description is described in the Appendix for keeping the whole paper short and perspicuous.

If the original object $S^{\prime}$ is rotated by a rotation matrix $R=\left[v_{1}, v_{2}, v_{3}\right]^{\mathrm{T}}$, the new symmetry axis of the rotated object $S$ will be $(0,0,1)^{\mathrm{T}}$. We may define a new signature function $f(X, Y, Z)$ representing the rotated object $S$ (see Fig. 5(b))

$$
f(X, Y, Z)=\left\{\begin{array}{l}
1: \text { if }(X, Y, Z) \text { is in } S \\
0: \text { if }(X, Y, Z) \text { is not in } S .
\end{array}\right.
$$

Now, equation (26) can be rewritten in a simpler form by the cylindrical coordinate system $r-\phi-Z$ (see Fig. 6).

$$
f(r, \phi, Z)=f\left(r, \phi+k\left(\frac{2 \pi}{N}\right), Z\right),
$$

where

$$
X=r \cos \phi ; \quad Y=r \sin \phi ; \quad Z=Z .
$$

Obviously, $f(r, \phi, Z)$ is a $1 \mathrm{D}$ periodic function of $\phi$ for any fixed $r$ and $Z$.

\subsection{Fourier transform of the object in $r-\phi-Z$ space}

Let $F(r, \omega, Z)$ denote the 1D Fourier transformation of the function $f(r, \phi, Z)$ with respect to the parameter $\phi$ for any fixed $r$ and $Z$. Similar to Section $2.2, f(r, \phi, Z)$ can be expanded by a Fourier series like this

$$
f(r, \phi, Z)=\sum_{s=-\infty}^{\infty} C_{s}(r, Z) e^{j s \omega_{0} \phi},
$$

where $\omega_{0} \equiv 2 \pi /(2 / \pi / N)=N$ is the fundamental frequency and

$$
C_{s}(r, Z) \equiv \frac{N}{2 \pi} \int_{\phi=-\pi / N}^{\pi / N} f(r, \phi, Z) e^{-j s \omega_{0} \phi} \mathrm{d} \phi .
$$

Therefore, the 1D Fourier transformation $F(r, \omega, Z)$ with respect to $\phi$ can be written as

$$
F(r, \omega, Z)=\sum_{s=-\infty}^{\infty} C_{s}(r, Z) \delta\left(\omega-s \omega_{0}\right) .
$$

Obviously, $F(r, \omega, Z)$ is zero except at the frequencies $\omega=s \omega_{0}$ for any fixed $r$ and $Z$. Once the fundamental frequency $\omega_{0}$ is determined, we can easily obtain the fold number $N$ of the ARS object.

\subsection{Our algorithm: finding the fold number $\mathrm{N}$}

For the computational efficiency, we first determine the maximum radius $r_{\max }$, minimum radius $r_{\min }$, maximum height $Z_{\max }$, and minimum height $Z_{\min }$ of the object $S$. The whole object is contained in the region $A \equiv\left\{(r, \phi, Z) \mid r_{\min } \leq r \leq r_{\max } ; 0 \leq \phi \leq 2 \pi ; Z_{\min } \leq Z \leq\right.$ $\left.Z_{\max }\right\}$. Without loss of generality, both of the maximum radius $r_{\max }$ and maximum height $Z_{\max }$ can be set to 1 by properly scaling the given ARS object

For the same reason for using digital hardware, the discrete Fourier transform (DFT) should be adopted to analyze the whole problem. So we may divide the whole region $A$ into $P \times Q \times N_{s}$ sub-blocks (see Fig. 7), where its $(p, q, k)$ th sub-block is denoted by $A_{p q k}(p=1$ to $P, q=1$ to $Q$, and $k=0$ to $N_{s}-1$ ). The three positive integers, $P, Q$ and $N_{s}$ separately represent the resolutions in the directions of $r, Z$, and $\phi$. Similar to Section 2, $P$ and $Q$ are the defined section numbers, and $N_{s}$ is the angular sampling number.

$$
\begin{array}{r}
A_{p q k}=\left\{(r, \phi, Z) \mid r_{p-1} \leq r \leq r_{p} ; \phi_{k} \leq \phi \leq \phi_{k+1}\right. \\
\left.Z_{q-1} \leq Z \leq Z_{q}\right\}
\end{array}
$$
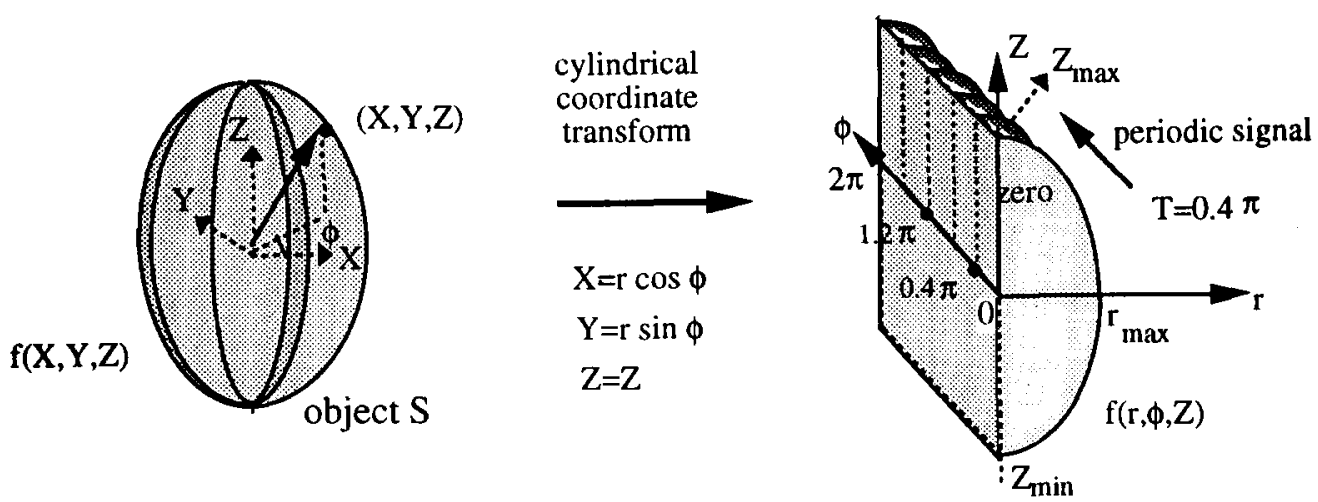

Fig. 6. Transform the $X Y Z$ coordinate system of the object $S$ to the cylindrical coordinate system $r \phi Z$. Here $f(r, \phi, Z)$ is a $1 \mathrm{D}$ periodic function of $\phi$ for any fixed $r$ and $Z$. 


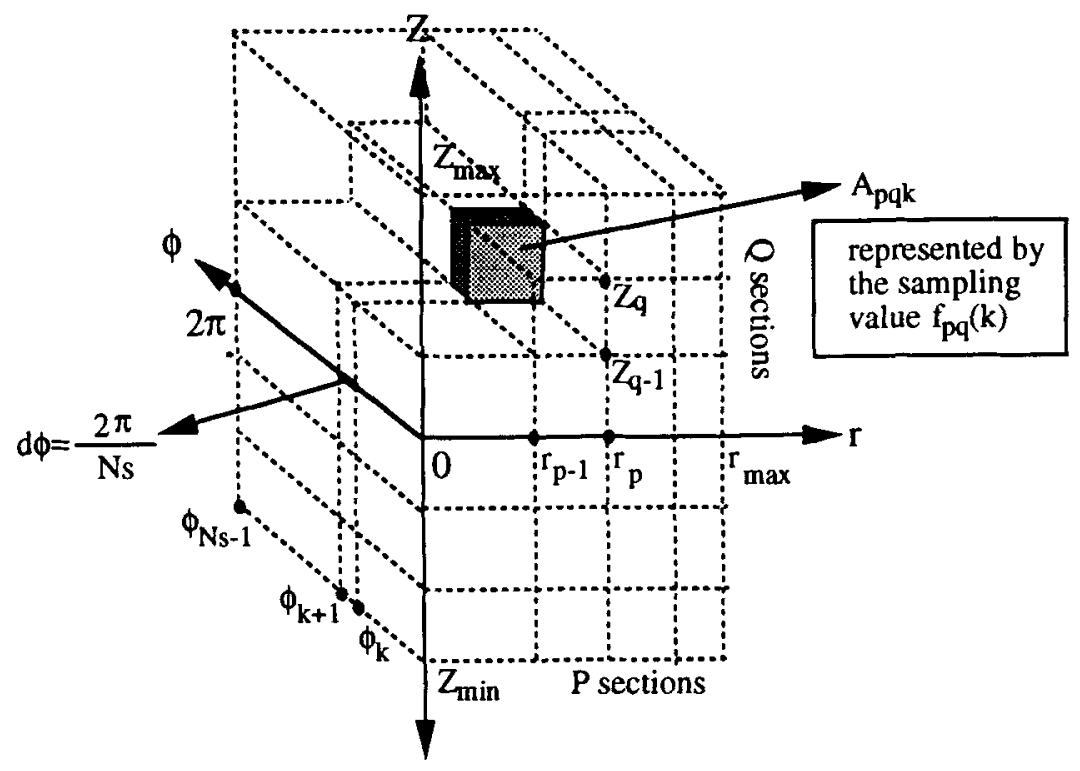

Fig. 7. The region $A$ which contains the whole object $S$ in the cylindrical coordinate system is divided into $P \cdot Q \cdot N_{s}$ small block regions, denoted by $A_{p q k}$. The average $f(r, \phi, Z)$ in the small block region $A_{p q k}$ is considered as a sampling value $f_{p q}(k)$.

where

$$
\begin{aligned}
& r_{p}=\sqrt{\left(\frac{p}{P}\right) r_{\max }^{2}+\left(\frac{P-p}{P}\right) r_{\min }^{2}} \\
& Z_{q}=\left(\frac{q}{Q}\right) Z_{\max }+\left(\frac{Q-q}{Q}\right) Z_{\min }
\end{aligned}
$$

and

$$
\phi_{k}=k \frac{2 \pi}{N_{s}}
$$

From the definitions in equations (36)-(39), every subblock, $A_{p q k}$, is of the same volume $\Delta$ defined as

volume of $A_{p q k}=\frac{\pi}{N_{s} P Q}\left(r_{\max }^{2}-r_{\min }^{2}\right)\left(Z_{\max }-Z_{\min }\right)=\Delta$

$$
\text { for all } p, q, k . \quad(40)
$$

Now we can define a value $f_{p q}(k)$ which can be considered as a sampling value of the sub-block $A_{p q k}$ (see Fig. 7)

$$
\begin{aligned}
f_{p q}(k) & =\frac{1}{\Delta} \iiint_{A_{p q k}} f(r, \phi, Z) r \mathrm{~d} r \mathrm{~d} \phi \mathrm{d} Z \\
& \approx \frac{2 \pi}{N_{s} \Delta_{Z_{q-1}}} \int_{Z_{q}}^{Z_{p_{p}-1}} \int_{r_{p}}^{r_{p}} f\left(r, \phi_{k}, Z\right) r \mathrm{~d} r \mathrm{~d} Z .
\end{aligned}
$$

The above equation is approximated because the angular sampling number $N_{s}$ is usually very large (e.g. 256).

Now we have $P Q 1 \mathrm{D}$ sequences $\left\{f_{p q}(0), \ldots, f_{p q}\left(N_{s}-1\right)\right\}$ for $p=1$ to $P$ and $q=1$ to $Q$. The DFTs of these 1D sequences can be defined as

$$
F_{p q}(k) \equiv \sum_{n=0}^{N_{s}-1} f_{p q}(n) e^{-j k\left(2 \pi n / N_{s}\right)}
$$

After substituting equations (33) and (41) into equation (42), we can obtain a new expression for $F_{p q}(k)$

$$
F_{p q}(k)=\sum_{s=-\infty}^{\infty} T_{p q, s} H_{k, s}
$$

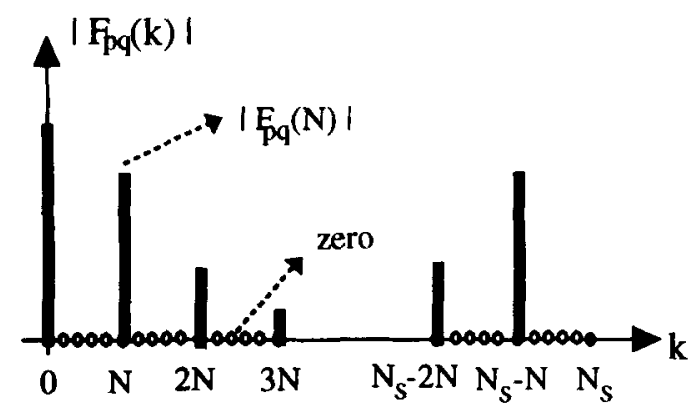

(a)

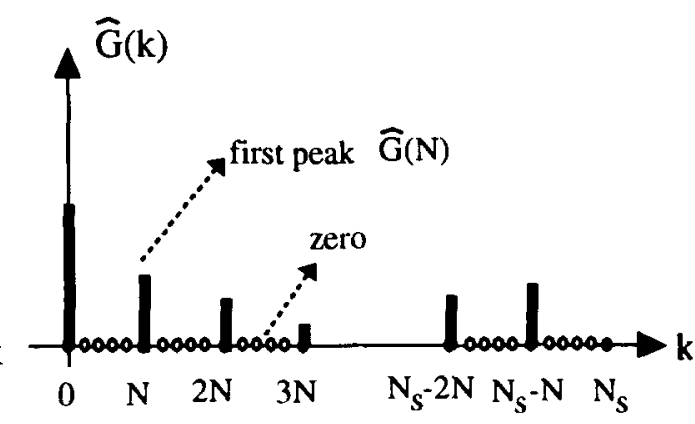

(b)

Fig. 8. The DFT of the 1D data sequence $\left\{f_{p q}(k)\right\}$, denoted by $\left\{F_{p q}(k)\right\}$. (b) The pseudo power spectrum function $\hat{G}(k) . k=0$ to $N_{s}-1$. If $N_{s}$ is large enough, $F_{p q}(k)$ and $\hat{G}(k)$ is zero except at the points $k=\mathrm{d} N$. 
where

$$
\begin{aligned}
H_{k, s} & \equiv \frac{1}{N_{s}} \sum_{n=0}^{N_{s}-1} e^{j(s N-k)\left(2 n \pi / N_{s}\right)} ; \\
T_{p q, s} & \equiv \frac{2 \pi}{\Delta} \int_{Z_{q-1}}^{Z_{q}} \int_{r_{p-1}}^{r_{p}} C_{s}(r, Z) r \mathrm{~d} r \mathrm{~d} Z .
\end{aligned}
$$

From equation (44), it is easy to observe that $H_{k, s}=1$ when $k=s N-a N_{s}$, and $H_{k, s}=0$ for any other $k$.

If the angular sampling number $N_{s}$ happens to be a multiple of the fold number $N, F_{p q}(k)$ can be simply written as

$F_{p q}(k)= \begin{cases}T_{p q, \mathrm{~d}}+\text { aliasing component. } & \text { if } k=\mathrm{d} N \\ 0 . & k=\text { otherwise }\end{cases}$

( $d$ is an integer). If $N_{s}$ is not a multiple of $N, F_{m}(k)$ can be simply written as

$F_{p q}(k)= \begin{cases}T_{p q, d}+\text { aliasing component. } & \text { if } k=\mathrm{d} N \\ \text { aliasing component. } & k=\text { otherwise }\end{cases}$

We now define a pseudo-power spectrum density function $\hat{G}(k)$ as

$$
\hat{G}(k)=\sum_{p=1}^{P} \sum_{q=1}^{Q}\left|F_{p q}(k)\right|^{2} .
$$

Detecting the location of the first peak of $\hat{G}(k)$ (i.e. $k=N)$ is equivalent to determining the fold number $N$ of the given ARS object. This function $\hat{G}(k)$, is especially called the $(P, Q)$-indicator in this paper.

\subsection{Normalization of object orientation}

After determining the fold number $N$, its $P Q$ corresponding DFT values, $F_{p q}(N)$ s, can be easily obtained. Similar to $2 \mathrm{D}$ case, we may define a complex value $C_{1}$ as the summation of all $F_{p q}(N) s$.

$$
C_{1}=\sum_{p=1}^{P} \sum_{q=1}^{Q} F_{p q}(N)=r_{c} e^{j \phi_{c}},
$$

where $0 \leq \phi_{c} \leq 2 \pi$ and $r_{c}>0$. Notice the angle $\theta_{c}$ will not be affected by the section numbers, $P$ and $Q$, because every sub-block $A_{p q k}$ has the same volume $\Delta$.

If the object $S$ is rotated around the $Z$-axis by an angle $\phi_{t}$, the rotated object can be represented by $f\left(r, \phi-\phi_{t}, Z\right)$. We can expand it by a Fourier series

$$
f\left(r, \phi-\phi_{t}, Z\right)=\sum_{s=-\infty}^{\infty}\left(C_{s}(r, Z) e^{-j s \omega_{0} \phi_{t}}\right) e^{j s \omega_{0} \phi} .
$$

Comparing equation (49) with equations (33) and (44), it is easy to observe that rotating the object around the $Z$-axis by an angle $\phi_{t}$ will induce a constant phase shift $\exp \left(-j s \omega_{0} \phi_{t}\right)$ to its sth Fourier coefficient $C_{s}(r, Z)$ and $T_{p q, s}$. If the aliasing components defined in equations (45) and (46) are negligible, all of the $F_{p q}(N) \mathrm{s}$ will have a constant phase shift $\exp \left(-j N \phi_{t}\right)$.

If the original ARS object $S$ can be rotated around the $Z$-axis by an approximate angle $\phi_{t}$ such that the calculated $C_{1}$ of the new object is a real positive value (phase $=0$ ), we say that the new rotated object $\hat{S}$ has been orientation-normalized (see Fig. 9). Such an ap-

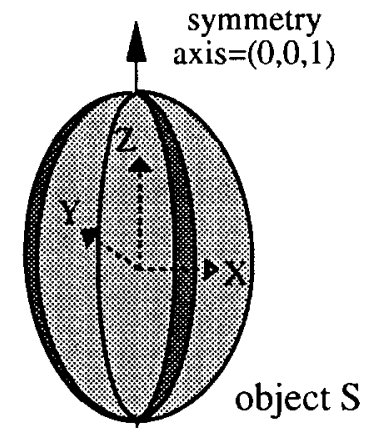

(not orientation-normalized)

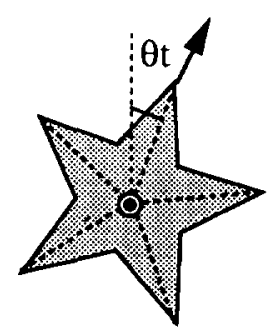

(Top-view of $\mathrm{S}$ )

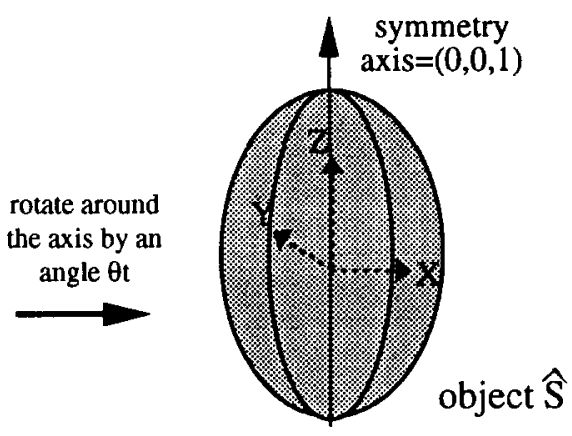

(orientation normalized)

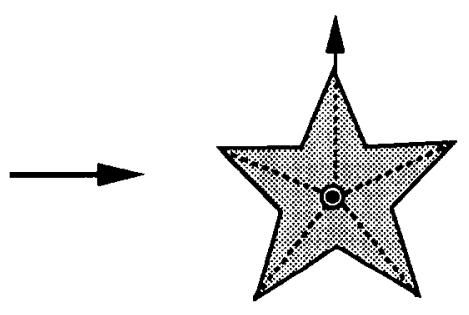

(top view of $\hat{\mathrm{S}}$ )

Fig. 9. Suitably rotate the original object $S$ around the $Z$-axis by an angle $\theta_{t}$ to normalize the orientation. 
propriate rotating angle $\theta_{s}$ is calculated by

$$
\phi_{t}=\frac{1}{N} \phi_{\mathrm{c}^{*}}
$$

If the amplitude of $C_{1}$ is too small, we may choose the one in all of the $F_{p q}(N)$ s (say $F_{a b}(N)$ ) which has the largest amplitude to replace the original $C_{1}$. Therefore,

$$
\phi_{t}=\frac{1}{N}\left(\arg \left(F_{a b}(N)\right)\right) .
$$

Now, the whole algorithm is completed.

\section{DISCUSSIONS}

In both of the 2D and 3D cases described in Section 2 and 3, our methods have similar processing steps: (1) change the original coordinate system $X Y$ (or $X Y Z$ ) into the polar coordinate system that can express more explicitly the periodicity of rotational symmetry; (2) reduce the original 2D shape (or 3D object) into several $1 \mathrm{D}$ data sequences; (3) calculate the DFTs of these 1D sequences; (4) determine the fold number $N$ by finding the first peak of a pseudo-power spectrum density function $\hat{G}(k)$; and (5) normalize the orientation of the $2 \mathrm{D}$ shape (or $3 \mathrm{D}$ object) by using the DFT values, $F_{m}(N) \mathrm{s}\left(\right.$ or $\left.F_{p q}(N) \mathrm{s}\right)$.

\subsection{How to choose the section number $\mathrm{M}$ ?}

At the second step, we say the original 2D (or 3D) data should be reduced to $M$ (or $P Q$ ) $1 D$ data sequences. How will the section number $M$ (or $P Q$ ) be chosen? Take the $2 \mathrm{D}$ case for example. We first consider an extreme case when $M=\infty$ and $N_{s}=\infty$. The pseudopower function, $\hat{G}(k)$, is now equal to the Fourier transform of the angular correlation function of the $2 D$ RS shape. Of no doubts, it is known to be the most reliable indicator to show the angular periodicity of a RS shape because no periodic information will be lost. On the other words, an indicator with a smaller section number is usually less reliable than that with a larger number because of more lost information.

To show this concept, let's see some special computergenerated $N$-fold RS symmetric shapes depicted in Fig. 10. The $M=1$ indicator (proposed by reference (16)) fails to determine their fold numbers because their reduced $1 D$ data sequences are all DC sequences, and all the periodicities are gone (see Fig. 10(e)). However, the $M=2$ (or larger) indicator can still successfully determine their fold numbers because some periodicities are reserved (see Fig. 10(f)). So the choice of $M$ is, in fact, a trade-off between the computational load and the reliability of the fold number's estimation. In real applications, the indicators whose section number $M$ is smaller than five are usually reliable enough for practical use.

A better choice of the section number $M$ depends on the complexity of the shape. For example, if a simple $2 \mathrm{D}$ shape whose boundary can be represented by a single-value function of the angle $\theta$ in the polar co- ordinate system, the $M=1$ indicator will be the best choice. It is because the original 2D shape can be completely reconstructed by its reduced 1D data sequence $\left\{f_{1}(k)\right\}$ and no periodic information will be lost. However, an indicator with larger $M$ is usually recommended when the shape is hollow, shell-like, or boundary-complicated. Similar rules can also be applied to the $3 \mathrm{D}$ case. If we have no prior information about the complexity of the shape, the indicators with the larger $M$ will be better.

\subsection{Threshold of the indicator $\hat{\mathrm{G}}(\mathrm{k})$}

To determine whether a periodic signal exists in $\hat{G}(k)$, an appropriate threshold must be specified first. Basically, this threshold depends on two factors: (1) magnitudes of the aliasing components; and (2) position errors of the boundary points. The first item depends on the sampling number $N_{s}$ and the angular periodic characters of the RS shapes (broad-banded or narrowbanded). The second item is often resulted from the quantization errors of the boundary points. Because it is hard to model these errors, it seems hard to obtain a general formula specifying the threshold value. Here we just approximate the threshold in a very coarse manner.

If the aliasing errors and quantization errors can provide a perturbation $\delta_{m}(k)$ to the sampling value $f_{m}(k)$ (similar to $f_{p q}(k)$ )

$$
\hat{f}_{m}(k)=f_{m}(k)+\delta_{m}(k),
$$

where the perturbation $\delta_{m}(k)$ is a random variable of normal distribution $N(0, \sigma)$ for $m=1$ to $M$ and $k=0$ to $N_{s}-1$. The DFT of $\left\{\delta_{m}(k)\right\}$ is denoted by $\left\{\Delta_{m}(k)\right\}$.

So we can derive an average bound of errors

$$
\begin{aligned}
& E\left(\sum_{m=1}^{M}\left|\Delta_{m}(k)\right|^{2}\right) \\
& =\sum_{m=1}^{M}\left[\sum_{n=0}^{N_{s}-1} \sum_{n^{\prime}=0}^{N_{s}-1} E\left(\delta_{m}(n) \delta_{m}\left(n^{\prime}\right)\right) \mathrm{e}^{-2 k \pi\left(n-n^{\prime}\right) / N_{s}}\right] \\
& =M N_{s} \sigma^{2}
\end{aligned}
$$

$E(\cdot)$ is the operator of ensemble average.

So the value $M N_{s} \sigma^{2}$ can be utilized to define an appropriate threshold. In the $3 \mathrm{D}$ case, the value is equal to $P Q N_{s} \sigma^{2}$ which can be derived by a similar procedure.

\section{EXPERIMENTS}

In this section, we design two parts of experiments for proving that the proposed algorithm can be successfully applied in the normalization of 2D RS shapes and 3D ARS objects.

\subsection{Experiments for $2 D$ shape}

In this subsection, two things will be proved: (1) the fold number $N$ can be correctly determined; and (2) The orientation of the given $2 \mathrm{D}$ shape can be success- 


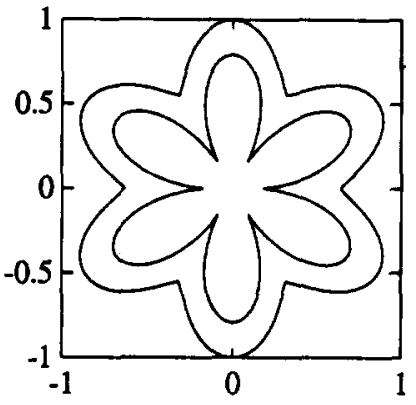

(a) The fold number $\mathrm{N}=6$

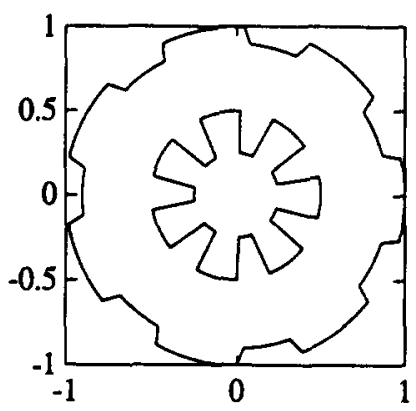

(c) The fold number $\mathrm{N}=7$

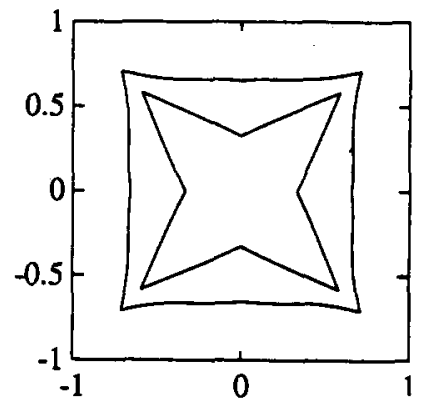

(b) The fold number $\mathrm{N}=4$

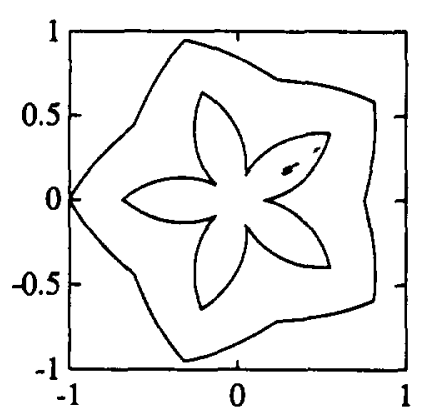

(d) The fold number $\mathrm{N}=5$
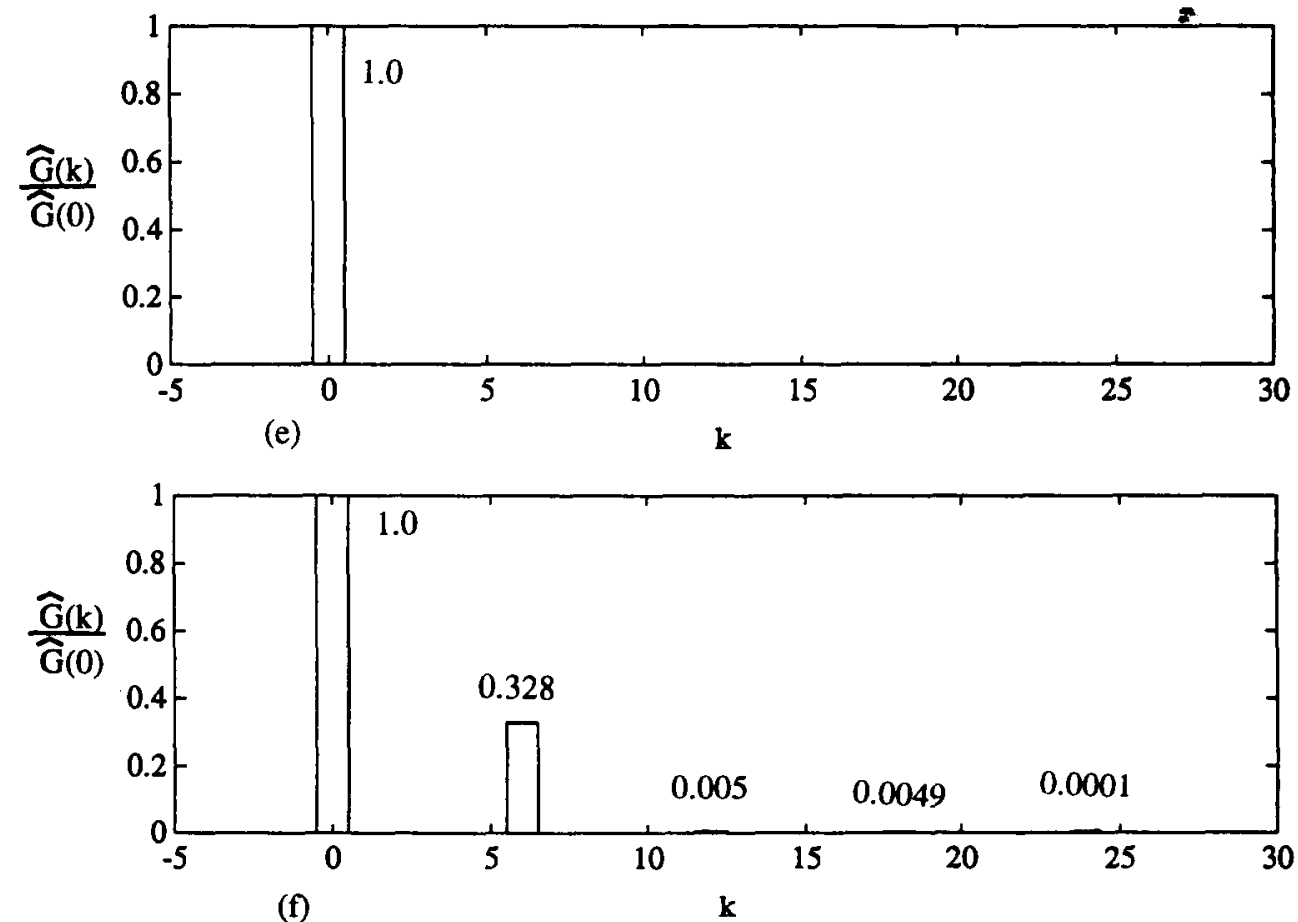

Fig. 10. (a)-(d) Are four special $N$-fold rotationally symmetric $2 \mathrm{D}$ shapes. The $M=1$ indicator fails to determine their fold numbers because all of their reduced $1 D$ data sequences are DC sequences. However, $M=2$ indicator is successful. (e) $M=1$ indicator. (f) $M=2$ indicator. 
fully normalized. The sampling number $N_{s}$ is chosen 256. It means a 256-point DFT is adopted in our experiments. Two computer-generated 2D shapes are tested here.

Let's see the first example shown in Fig. 11. It is a simple, of no inside holes, five-fold RS shape. Its outer boundary contour can be represented by a single-value function of the angle $\theta$. A large part of RS shapes seen in daily life belong to this kind. The $M=1$ indicator $\hat{G}(k)$ is chosen to determine the fold number $N$. Fig- ure 11(a) and (c) show two arbitrarily-oriented shapes $S_{1}$ and $S_{2}$. Figure 11(b) and (d) are separately their normalized poses, $\hat{S}_{1}$ and $\hat{S}_{2}$. Notice that these two normalized shapes are almost identical. Figure 11(e) shows the result of the $M=1$ indicator $\hat{G}(k)$. It is zero except at the points $k=5 q$ ( $q$ is an integer). So the fold number $N(=5)$ is correctly determined.

The second example shown in Fig. 12 test a more complicated shape $(N=4)$. The $M=3$ indicator is used as a more reliable indicator than that of $M=1$.

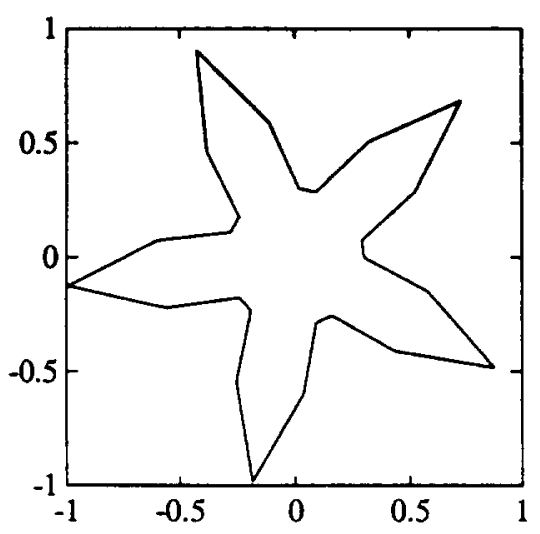

(a) The given shape $S_{1}$

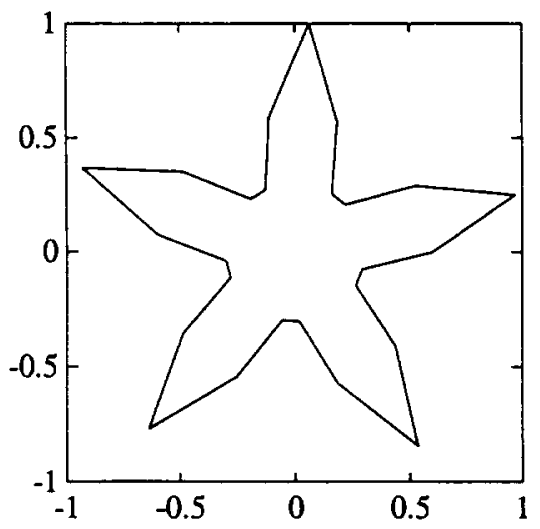

(c) The given shape $\mathrm{S}_{2}$

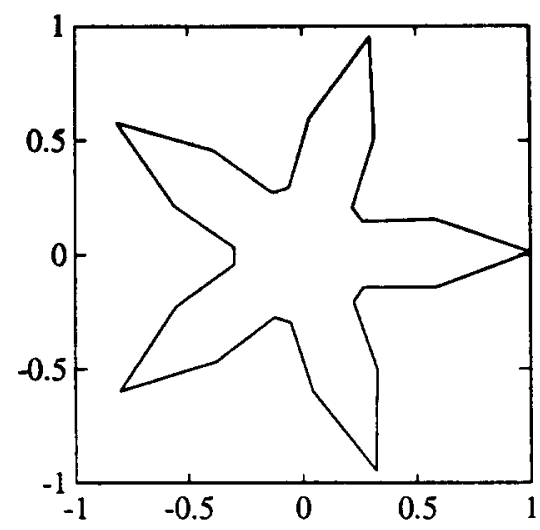

(b) The orientation-normalized shape $\hat{S}$,

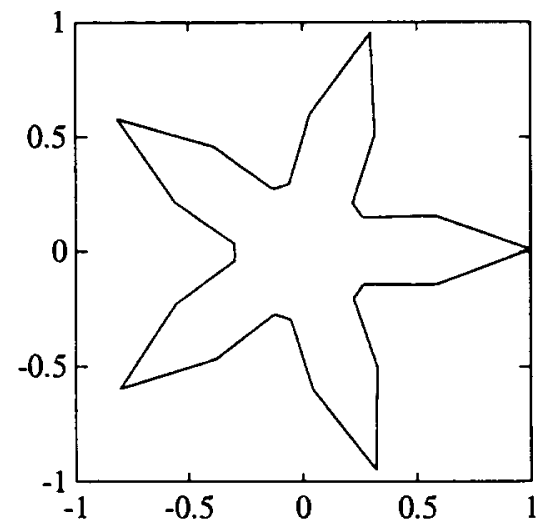

(d) The orientation-normalized shape $\widehat{S}_{2}$

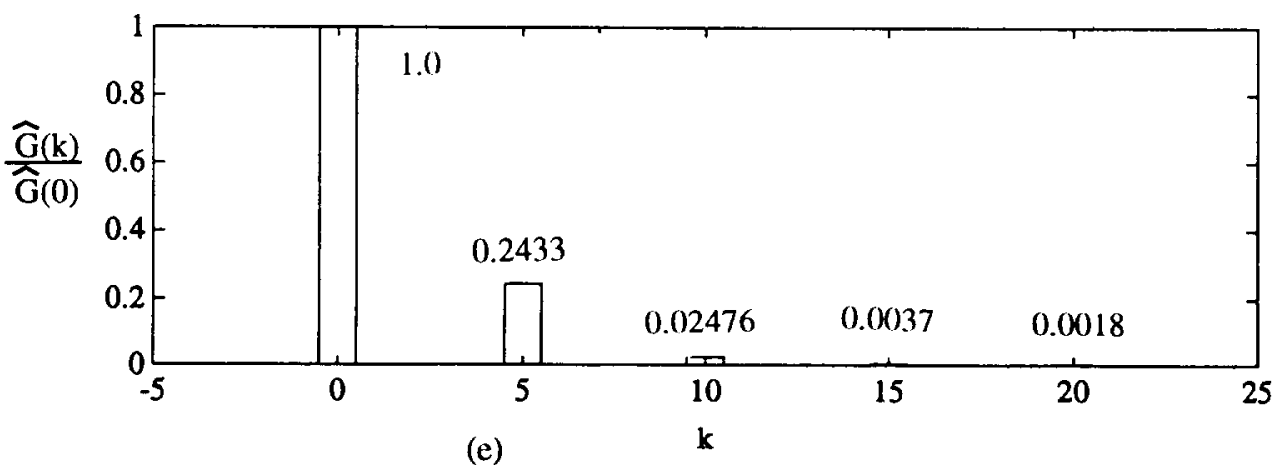

Fig. 11. (a) A shape with an arbitrarily-chosen orientation. (b) The normalized shape of (a). (c) Another shape with an arbitrarily-chosen orientation. (d) The normalized shape of (c). Notice that (b) and (d) are almost identical. (e) The $M=1$ indicator. 


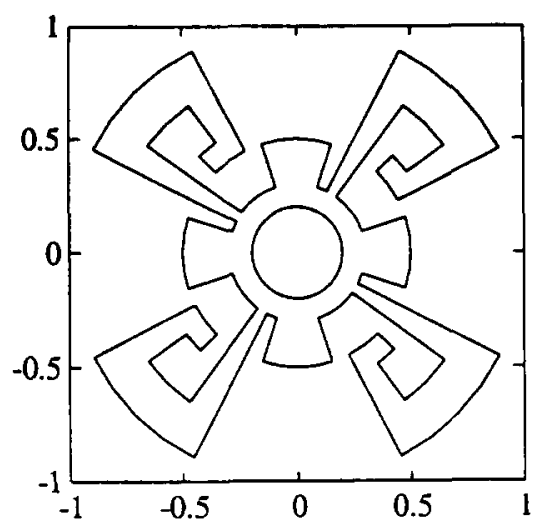

(a) The given shape $S_{1}$

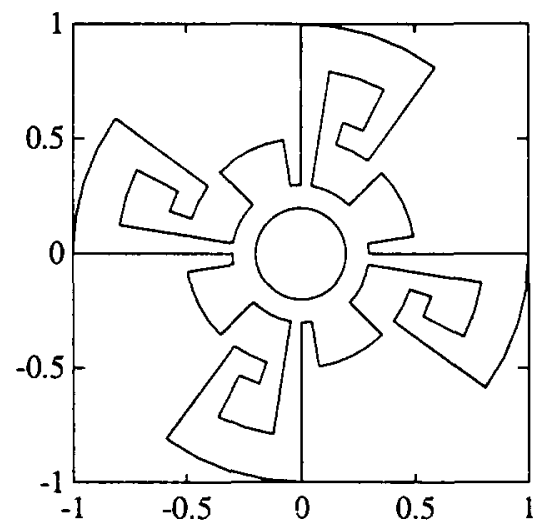

(c) The given shape $S_{2}$

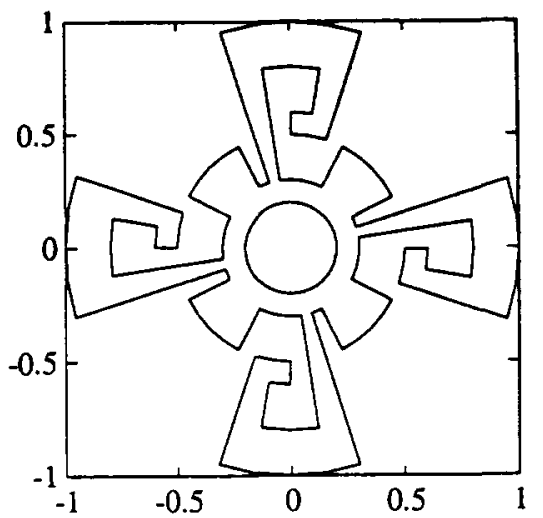

(b) The orientation-normalized shape $\widehat{S}_{1}$

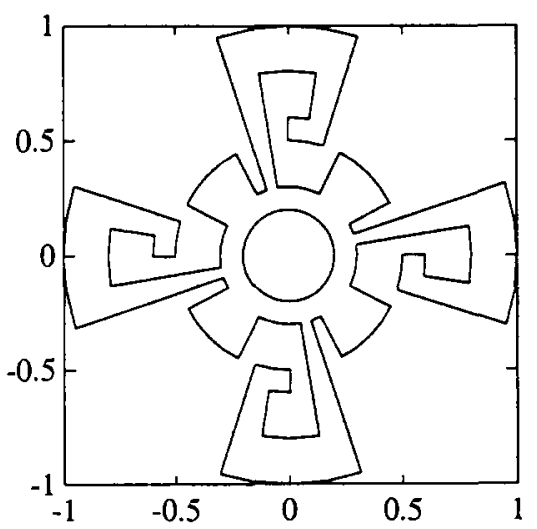

(d) The orientation-normalized shape $\hat{S}_{2}$

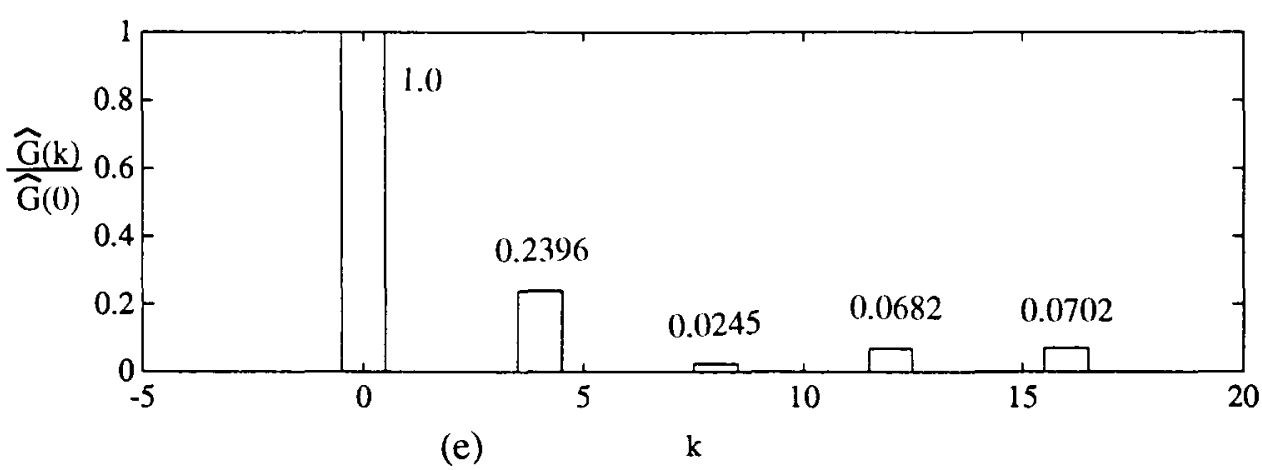

Fig. 12. (a) A shape with an arbitrarily-chosen orientation. (b) The normalized shape of (a). (c) Another shape with an arbitrarily-chosen orientation. (d) The normalized shape of (c). Notice that (b) and (d) are almost identical. (e) The $M=3$ indicator.

Its fold number $(=4)$ and orientations are successfully determined and normalized, too.

\subsection{Experiments for $3 D$ object}

Similar to the previous experiments, two things will be proved here: (1) the symmetry axis $n$ and the fold number $N$ of a given ARS 3D object can be correctly determined; and (2) Its orientation can be successfully normalized. The sampling number $N_{s}$ is also chosen 256. Two computer-generated $3 \mathrm{D}$ objects are tested.
In the first example, a five-fold object which looks like a five-leaf fruit is tested and shown in Fig. 13. Figure 13(a) and (c) show two arbitrarily-chosen poses, $S_{1}^{\prime}$ and $S_{2}^{\prime}$, of the ARS 3D objects. Figure 13(b) and (d) show their normalized objects. Notice that these two normalized objects, $\hat{S}_{1}$ and $\hat{S}_{2}$, are almost identical. Figure $13(\mathrm{e})$ shows the result of the $(1,1)$-indicator. Its fold number $N(=5)$ can be correctly determined. The three eigenvalues of the dispersion matrix of the object $S_{1}^{\prime}$ (or $S_{2}^{\prime}$ ) are $\{0.5426,0.5426,0.7667\}$. We can see that two of these eigenvalues are equal. The detected sym- 


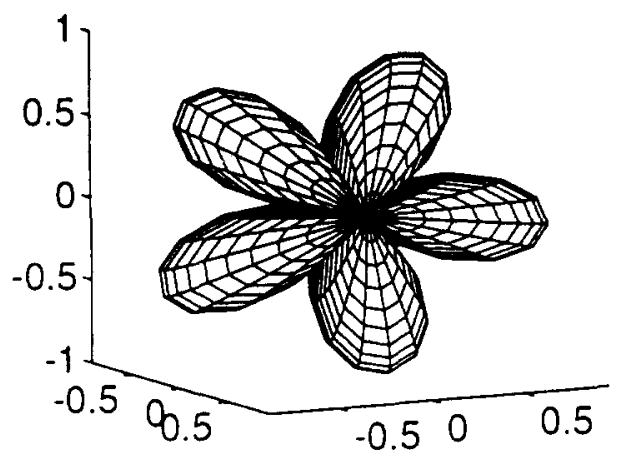

(a)

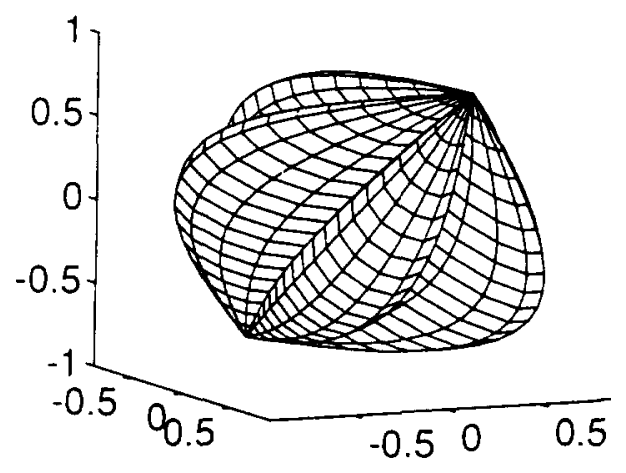

(c)

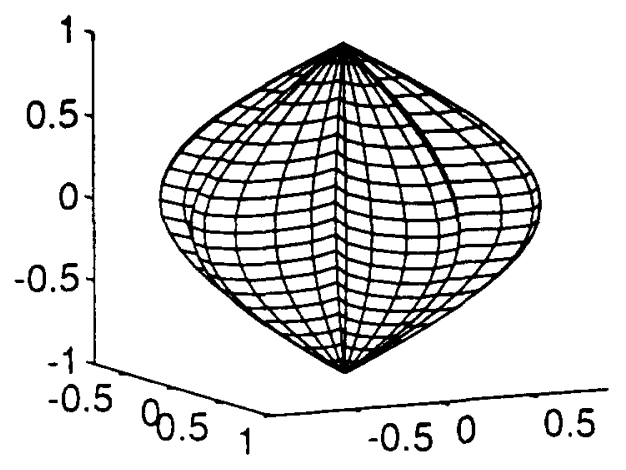

(b)

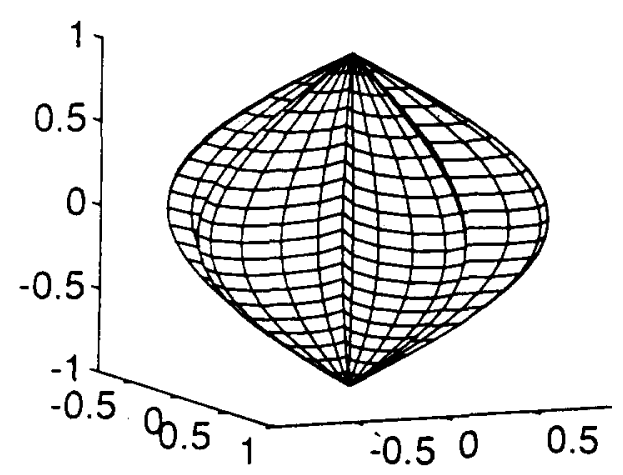

(d)

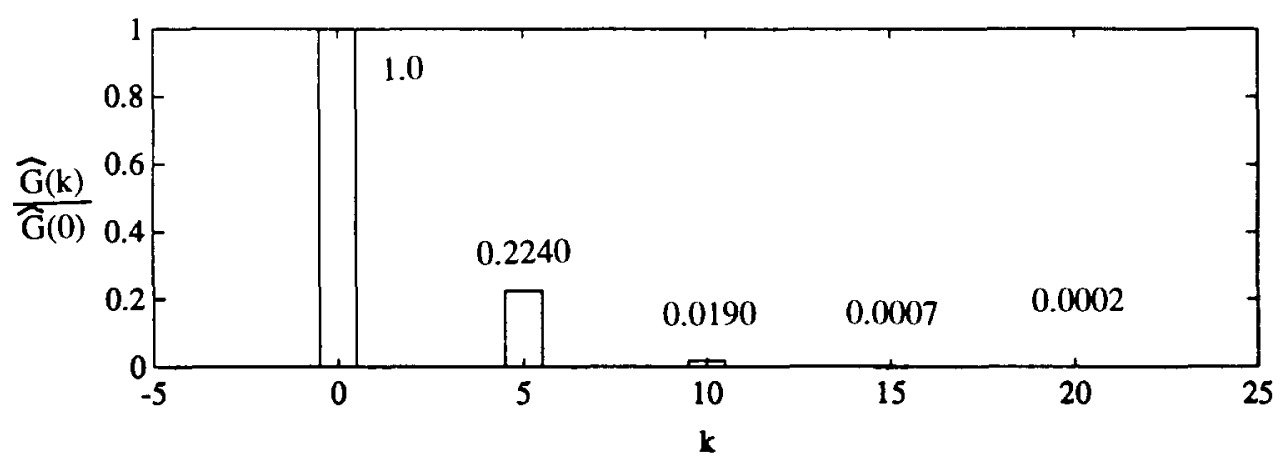

(e)

Fig. 13. (a) An object with an arbitrarily-chosen orientation. (b) The normalized object of (a). (c) Another object with an arbitrarily-chosen orientation. (d) The normalized object of (c). Notice that (b) and (d) are almost identical. (e) The $(1,1)$-indicator.

metry axes of the objects $S_{1}^{\prime}$ and $S_{2}^{\prime}$ are $(0.9319,-0.3346$, $0.1402)^{\mathrm{T}}$ and $(0.4980,0.3324,0.8009)^{\mathrm{T}}$, just the same as the true directions we previously assign.

A three-fold object is tested in the second example. This object can be divided into three parts: (1) the upper part is three-fold; (2) the middle part is a conical surface; and (3) the lower part is six-fold. Of course, it is still a three-fold ARS object. All the results are shown in Fig. 14 and their explanations are similar to that in Fig. 13. The $(3,3)$-indicator is used in this example. Three eigenvalues of the dispersion matrix of the object $S_{1}^{\prime}\left(S_{2}^{\prime}\right)$ are $\{0.2772,0.2772,0.5625\}$. We can see that two of these eigenvalues are equal. The detected symmetry axes of the objects $S_{1}^{\prime}$ and $S_{2}^{\prime}$ are $(0.1955$, $-0.5185,0.8324)^{\mathrm{T}}$ and $(0.5176,0.7166,0.4674)^{\mathrm{T}}$, just the same as the true directions we previously assign. 


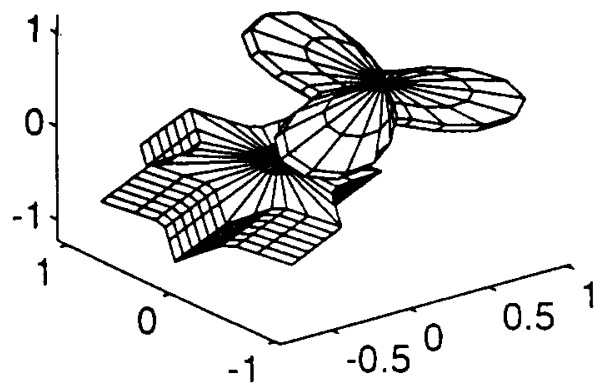

(a)

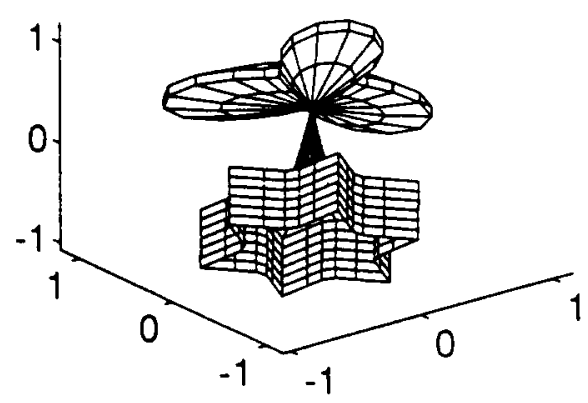

(c)

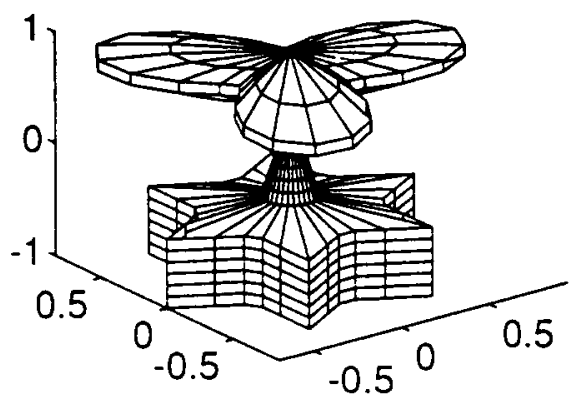

(b)

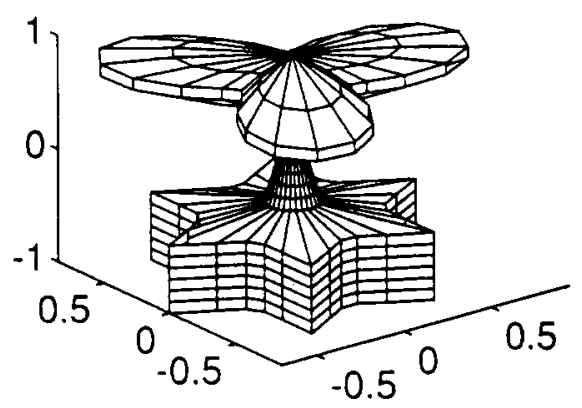

(d)

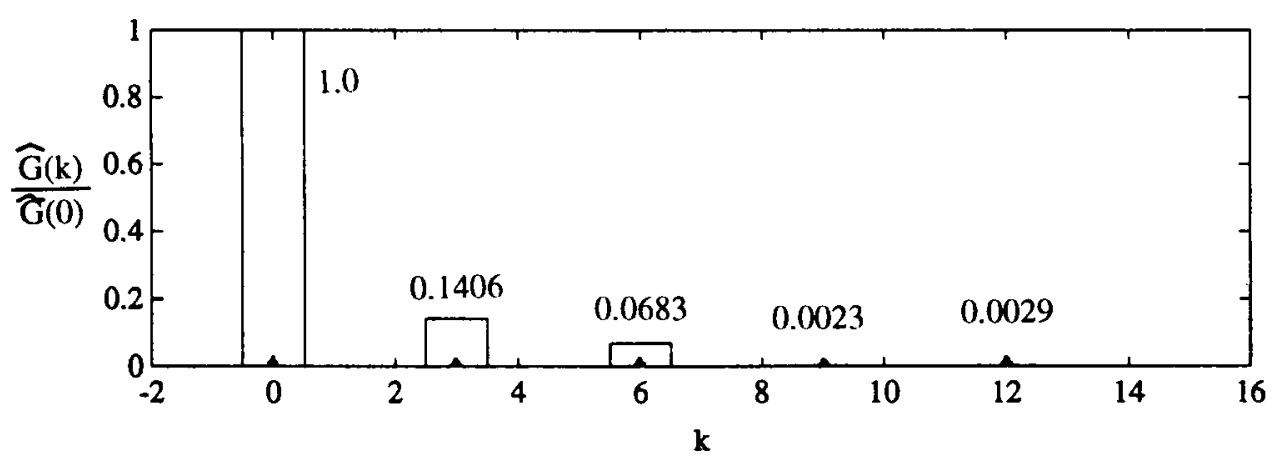

(e)

Fig. 14. (a) An object with an arbitrarily-chosen orientation. (b) The normalized object of (a). (c) Another object with an arbitrarily-chosen orientation. (d) The normalized object of (c). Notice that (b) and (d) are almost identical. (e) The $(3,3)$-indicator.

\section{CONCLUSION}

In this paper, we propose an efficient and reliable new method for fold number determination and orientation normalization of a $N$-fold rotationally-symmetric $2 \mathrm{D}$ shape. The 2D shapes are not constrained to simple closed contours, and they can be hollow or any complex shapes. Basically, our method reduces the original 2D data function into $M$ 1D data sequences (not only one). The DFTs of these 1D sequences are then utilized in determining the fold number and normalizing the shape's orientation.
This method is also extended to solve a $N$-fold axial rotationally symmetric 3D object. Its symmetry axis $\mathbf{n}$ is first determined by finding the corresponding eigenvector $v_{3}$ of the distinct eigenvalue $\lambda_{3}$ of the object's dispersion matrix $M$. Similar to the $2 \mathrm{D}$ case, the $3 \mathrm{D}$ data function is reduced to $P Q 1 D$ data sequences. Their DFTs are then utilized in determining the fold number and normalizing the orientation of the object.

The choice of the section number $M$ (or $P$ and $Q$ ) is a trade-off between the computational load and the reliability of the determined fold number $N$. Larger section numbers $(M$ or $(P, Q))$ usually implies a heavier 
computation load and a more reliable fold number estimation. If the shape is complex or shell-like, larger section number is recommended. If, on the contrary, the test shape is a simple closed contour which can be represented by a single-value function of parameter $\theta$, smaller section ( $M=1)$ is recommended.

There are two most obvious advantages in our new method: (1) it prevents the high computation load and the difficulty in normalizing the shape's orientation occurred in the old methods using full matching or correlation functions; and (2) The weakness introduced by the old methods using data reduction (from $2 \mathrm{D}$ data to $1 \mathrm{D}$ data) is remedied.

\section{REFERENCES}

1. A. Rosenfeld and A. C. Kak, Digital Picture Processing, Vol. II, pp. 289-290, Academic Press, New York (1982).

2. A. Taza and C. Y. Suen, Discrimination of planar shapes using shape matrices, IEEE Trans. Syst. Man Cybern. 19(5), 1281-1289 (September/October 1989).

3. S. A. Friedberg, Finding axes of skewed symmetry, Comput. Vision Graphics Image Process. 34, 138-155 (1986).

4. M. J. Atallah, On symmetry detection, IEEE Trans. Comput. 34, 663-666 (1985).

5. G. Marola, On the detection of the axes of symmetry of the symmetric and almost symmetric planar image, IEEE Trans. Pattern Analysis Mach. Intell. PAMI-11, 104-108 (1989).

6. B. B. Chaudhuri and D. D. Majumder, Recognition and fuzzy description of sides and symmetries of figures by computer, Int. J. System Science 11, 1435-1455 (1980).

7. C. T. Zahn and R.S. Roskies, Fourier descriptors for plane closed curves IEEE Trans. Comput. C-21, 269-281 (March 1972).

8. T. P. Wallace and P. A. Wintz, An efficient 3D aircraft recognition algorithm using normalized Fourier descriptor, Comput. Graphics Image Process. 13, 99-126 (1980).

9. T. R. Crimmins, A complete set of Fourier descriptors for 2D shapes, IEEE Trans. Syst. Man Cybern. SMC-7, 848855 (1982).

10. Y.S. Abu-Mostafa and D. Psaltis, Image normalization by complex moments, IEEE Trans. Pattern Analysis Mach Intell. PAMI-7, 46-55 (1985).

11. W. A. Perkins, A model based vision system for industrial parts, IEEE Trans. Comput. C-27, 126-143 (1978).

12. P. T. Highnam, Optimal algorithm for finding the symmetries of a planar point set, Information Process. Lett. 22(5), 219-222 (1986).

13. Wen-Hsiang Tsai and Sheng-Lin Chou, Detection of generalized principal axes in rotationally symmetric shapes, Pattern Recognition 24(2), 95-104 (1991).

14. Jin-Jang Leou and Wen-Hsiang Tsai, Automatic rotational symmetry determination for shape analysis, Pattern Recognition 20(6), 571-582 (1987).
15. Ja-Chen Lin, Sheng-Lin Chou and Wen-Hsiang Tsai, Detection of rotationally symmetric shape orientations by fold-invariant shape-specific points, Pattern Recognition 25(5), 473-482 (1992).

16. Soo-Chang Pei and Chao-nan Lin, Normalization of rotationally symmetric shapes for pattern recognition, Pattern Recognition 25(9), 913-920 (1992).

\section{APPENDIX}

From the proof of Tsai's paper ${ }^{(13-16)}$, we know the dispersion matrix of a $N$-fold rotationally symmetric $2 \mathrm{D}$ shape $(N>=3)$ is equal to a scaled identity matrix $(=a \mathbf{l})$. Now, to a $N$-fold ARS 3D object, we want to know what kind of special characters may exist in its dispersion matrix.

Without loss of generality, let's consider a $N$-fold ARS 3D object $S$ whose symmetry axis $n$ is equal to $(0,0,1)^{\mathrm{T}}$. Its dispersion matrix is still defined as

$$
M=\left[\begin{array}{lll}
m_{200} & m_{110} & m_{101} \\
m_{110} & m_{020} & m_{011} \\
m_{101} & m_{011} & m_{002}
\end{array}\right],
$$

where

$$
m_{i j k}=\frac{1}{|S|^{i+j+k}} \int_{Z_{\min }}^{Z_{\max }}\left(\iint_{S(Z)} f^{\prime}(X, Y, Z) X^{i} Y^{j} \mathrm{~d} X \mathrm{~d} Y\right) Z^{k} \mathrm{~d} Z .
$$

$S(Z)$ is a cross-section parallel to the $X-Y$ plane at height $Z$. $f(X, Y, Z)$ is a signature function whose value is 0 or $1 .|S|$ is the volume of the object $S$.

Because the symmetry axis $\mathbf{n}$ is just equal to the $Z$-axis of the coordinate system, every cross-section $S(Z)$ is at least $N$-fold rotationally-symmetric (or $k N$-fold, $k$ is a positive integer). The centroid of each cross-section slice must be located at $(0,0, Z)^{\mathrm{T}}$. Therefore, $m_{101}=m_{011}=0$. On the other side, we know the dispersion matrix of a $N$-fold RS 2D shape is equal to a scaled-identity matrix $(=a \mathbf{I})$. From equation (A2), we have

$$
\left[\begin{array}{ll}
m_{100} & m_{110} \\
m_{110} & m_{020}
\end{array}\right]=K \mathbf{I}
$$

where $K$ is a constant, $I$ is a $2 \times 2$ identity matrix.

Equation (A1) can be written in a simpler form

$$
M=\left[\begin{array}{ccc}
K & 0 & 0 \\
0 & K & 0 \\
0 & 0 & m_{002}
\end{array}\right]
$$

Obviously, $M$ has two same eigenvalues $\left(\lambda_{1}=\lambda_{2}=K\right)$ and one distinct eigenvalue $\left(\lambda_{3}=m_{002}\right)$. The corresponding eigenvector of $\lambda_{3}$ is $\hat{\mathbf{z}}=(0,0,1)^{\mathrm{T}}$, that is just the symmetry axis we defined earlier. Rotating the object $S$ by any rotation matrix $R$ will not change the eigenvalues. The eigenvector corresponding to the new distinct eigenvalue must be equal to $(R 乏)$, the new symmetry axis. It completes the whole proof.

\footnotetext{
About the Author-Soo-Chang Pel was born in Soo-Auo, Taiwan, China on 20 February 1949. He received the B.S. degree from National Taiwan University in 1970 and the M.S. and Ph.D. degree from the University of California, Santa Barbara in 1972 and 1975, respectively, all the electrical engineering.

He was an engineering officer in the Chinese Navy Shipyard at Peng Fu Island from 1970 to 1971 and a Research Assistant at the University of California, Santa Barbara from 1971 to 1975 . He was Professor and Chairman in the Department of Electrical Engineering at Tatung Institute of Technology from 1981 to 1983. Presently, he is the professor of Department of Electrical Engineering at National Taiwan University. His research interests include digital signal processing, digital picture processing, optical information processing, laser and holography.

Dr Pei is a member of the IEEE, Eta Keppa Nu and the Optical Society of America.
} 
About the Author-Lin-Gwo Liou was born in Taiwan. He received the B.S. degree from the National Chiao Tung University (N.C.T.U.) in Taiwan in 1989. Now, he is at the National Taiwan University (N.T.U.) studying for a Ph.D. degree. His research interests include motion image analysis, methods for 3D object reconstruction, pattern recognition in image application. 